\title{
Effects of long-term treatment for obstructive sleep apnea on pulse wave velocity
}

\author{
Tsunenori Saito ${ }^{1}$, Tsunehiro Saito ${ }^{2}$, Shigeyuki Sugiyama ${ }^{2}$, Kuniya Asai ${ }^{1}$, Masahiro Yasutake ${ }^{1}$ and \\ Kyoichi Mizuno ${ }^{1}$
}

Continuous positive airway pressure (CPAP) treatment improves endothelial function and sympathetic activity in patients with obstructive sleep apnea (OSA). However, the long-term effects of CPAP on pulse wave velocity (PWV), which reflects arterial stiffness that is associated with cardiovascular events, have not been evaluated in OSA patients with or without hypertension (HT). In this study, 212 male OSA patients who had been receiving CPAP treatment for 2 years and were divided into two groups, those with HT $(n=114)$ and those without $(n=98)$, were studied. In both HT and normotensive (NT) patients, PWV decreased significantly over the first 6 months of treatment $(P=0.005$ and 0.010 , respectively), before increasing gradually from 6 to 24 months. Body mass index (BMI), body weight, heart rate (HR), systolic blood pressure (SBP) and diastolic blood pressure (DBP) levels decreased significantly in the HT group over the 2 years of CPAP treatment $(P<0.001$ for all). In contrast, only HR decreased significantly in the NT group over the 2 years of treatment $(P<0.001)$. Multivariate regression analysis revealed that age $(P=0.008)$, decreases in DBP $(P<0.001)$ and HR $(P<0.001)$ and higher initial levels of serum high-density lipoprotein-cholesterol $(P=0.040)$ were independent factors related to changes in PWV over the 2 years of CPAP treatment in all patients. In conclusion, we found a significant decrease in PWV in both NT and HT patients after 6 months of CPAP treatment. In HT patients, long-term CPAP treatment significantly decreases blood pressure, which may contribute to explain the PWV improvement.

Hypertension Research (2010) 33, 844-849; doi:10.1038/hr.2010.77; published online 24 June 2010

Keywords: continuous positive airway pressure; heart rate; obstructive sleep apnea; pulse wave velocity

\section{INTRODUCTION}

Obstructive sleep apnea (OSA) is associated with cardiovascular and cerebrovascular mortality and morbidity, including hypertension (HT), coronary artery disease, heart failure and stroke. ${ }^{1,2}$ Atherosclerosis is a progressive disease that is also a pivotal risk factor for these cardiovascular disorders. OSA induces systemic HT, inflammation, ${ }^{3}$ endothelial dysfunction ${ }^{4}$ and disturbances in sympathetic activity, ${ }^{5}$ all of which are known to accelerate the development of atherosclerosis. It has been shown that continuous positive airway pressure (CPAP) treatment improves these risk factors for atherosclerosis. ${ }^{3-6}$ Arterial stiffness, an indicator of worsening atherosclerosis, has been shown in OSA patients, with the degree of arterial stiffness correlated with the severity of OSA. ${ }^{7,8}$ Pulse wave velocity (PWV) reflects arterial stiffness associated with cardiac events, and thus the severity of atherosclerosis. ${ }^{9}$ In addition, PWV is an independent predictor of cardiovascular mortality. ${ }^{10} \mathrm{~A}$ few recent studies showed that CPAP effectively improved PWV when used for short periods of time. ${ }^{11-15}$ However, the long-term effects of CPAP on PWV remain unclear. Previous studies of the short-term effects of CPAP on PWV were performed in normotensive (NT) OSA patients; thus, the effects of CPAP on PWV in patients with HT are unknown. The aims of this study were to evaluate the longterm effects of CPAP on PWV in both NT and HT OSA patients and to clarify the factors contributing to changes in PWV in these patients.

\section{METHODS}

Patient population

The subjects of this study consisted of 223 men who had been treated for OSA, which is a condition characterized by repeated episodes of upper airway obstruction during sleep, and $>30$ events of apnea and hypopnea per hour of sleep, with CPAP over a 2-year period, at the Good-Sleep Clinic from November 2004 to September 2006. Polysomnography was used for the diagnosis of OSA. Patients were followed up for at least 2 years prospectively. In all, 11 patients were subsequently excluded from the study because of poor treatment compliance, resulting in a final sample size of 212. All patients were middle aged (mean ( \pm s.d.) age $45.0 \pm 9.3$ years); 114 patients $(54 \%)$ had HT, $32(15 \%)$ had diabetes, $191(90 \%)$ had dyslipidemia and $58(27 \%)$ had hyperuricemia. In this study, patients were defined as having diabetes if they exhibited hyperglycemia (fasting glucose $>126 \mathrm{mg}$ per $100 \mathrm{ml}$ or plasma glucose $>200 \mathrm{mg}$ per $100 \mathrm{ml} 2 \mathrm{~h}$ after oral administration of the glucose load) or were being treated for diabetes mellitus. Patients were classified as having dyslipidemia if they exhibited hypertriglyceridemia ( $T G \geqslant 150 \mathrm{mg}$ per $100 \mathrm{ml}$ ), 
hypercholesterolemia (total cholesterol $\geqslant 220 \mathrm{mg}$ per $100 \mathrm{ml}$ ), or had low HDL-cholesterol levels (HDL $<40 \mathrm{mg}$ per $100 \mathrm{ml}$ ) or were being treated for dyslipidemia. Finally, patients were determined to be hyperuricemic if their serum uric acid levels were $>7 \mathrm{mg}$ per $100 \mathrm{ml}$ or they were being treated for hyperuricemia.

Patients were divided into two groups, those with HT (HT group; $n=114$ ) and those without (NT group; $n=98$ ). Normotension was defined as systolic blood pressure (SBP) $<140 \mathrm{~mm} \mathrm{Hg}$ and diastolic pressure (DBP) $<90 \mathrm{~mm} \mathrm{Hg}$; in addition, patients in the NT group were not taking any antihypertensive medication.

Written informed consent was obtained from all patients before their inclusion in the study.

\section{Measurement of blood pressure}

Blood pressure measurements were determined on separate occasions by more than three readings of systolic and diastolic (Korotkoff phases I and V, respectively) blood pressures obtained at 5 -min intervals by using a mercury sphygmomanometer, after patients had been seated for more than $10 \mathrm{~min}$ in a quiet room with a stable temperature and luminosity.

\section{CPAP treatment}

Patients were autotitrated and treated using an automated CPAP device (S8 Escape; ResMed, Sydney, NSW, Australia). Compliance with CPAP treatment was monitored every night over the 2 -year period, with adequate compliance prospectively defined as a mean of $5 \mathrm{~h}$ CPAP per night.

\section{Measurement of PWV}

PWV was measured after at least a 5-min rest. Brachial-ankle PWV was detected using a volume plethysmogram (Form/ABI; Colin, Komaki, Japan). The validity and reproducibility of this technique for measuring PWV have been described previously. ${ }^{16}$

\section{Measurement of percentage body fat (PBF)}

PBF was calculated from the Tanita multiple regression model (BC 118D; Tanita Corporation, Tokyo, Japan), using patient height, weight and age in a sex-specific equation.

\section{Laboratory measurements}

Fasting blood samples were obtained from all patients at baseline. Serum levels of total cholesterol, high-density lipoprotein-cholesterol (HDL-C), triacylglycerol and fasting blood sugar were measured using standard enzymatic methods (Falco Biosystems, Tokyo, Japan).

\section{Data analysis}

Body mass index (BMI), PBF, heart rate (HR), SBP, DBP and PWV were measured before and after 6,12 and 24 months of CPAP treatment. Results for the apnea-hypopnea index (calculated as the number of apnea and hypopnea events per hour of sleep), Epworth sleepiness scale, ${ }^{17}$ lowest oxygen saturation during sleep study and blood chemistry data were evaluated before treatment. Apnea was defined as complete cessation of airflow for at least $10 \mathrm{~s}$, whereas hypopnea was defined as a significant reduction $(<50 \%)$ in respiratory signals for at least $10 \mathrm{~s}^{8}{ }^{8}$ Data obtained at admission were compared between the NT and HT groups using Welch's $t$-test. Changes at 6 or 24 months are expressed as $\Delta$ parameter6 and $\Delta$ parameter24, respectively, where $\Delta$ parameter was calculated as follows:

\section{$\Delta$ Parameter $=($ Baseline value $)-($ Value after CPAP treatment $)$}

Changes in parameters (i.e., body weight, BMI, PBF, HR, SBP, DBP and PWV) were compared using two-way repeated-measures analysis of variance (ANOVA) over time and by Tukey's test at specific time points. Multiple regression analysis was applied to determine factors correlated with changes in PWV after 24 months of CPAP treatment for the total population. The correlation coefficient between $\triangle \mathrm{PWV} 6$ and $\triangle \mathrm{PWV} 24$ was calculated to determine the association between decreases in PWV after 6 and 24 months of CPAP treatment. Statistical analyses were performed using the SPSS software package
(SPSS, Chicago, IL, USA) and $P<0.05$ was considered significant. All data are expressed as the mean \pm s.d.

\section{RESULTS}

\section{Patient characteristics}

The baseline clinical characteristics of patients in the NT and HT groups are summarized in Table 1. Patients in the HT group were older $(P=0.002)$ and had a higher BMI $(P=0.037)$, PWV $(P<0.001)$, apnea-hypopnea index $(P<0.001)$, CPAP pressure $(P<0.001)$, serum triacylglycerol $(P=0.042)$ and prevalence of statin use $(P<0.001)$ than those in the NT group.

\section{Effects of CPAP}

The effects of CPAP treatment on the various parameters measured in this study are summarized in Table 2, Figures 1 and 2. Across the total study cohort, CPAP treatment for 2 years generally decreased BMI $(P<0.001)$, body weight $(P=0.004)$, PBF $(P<0.001)$, SBP $(P=0.003)$, DBP $(P=0.004)$ and HR $(P<0.001)$ (Table 2$)$. There was a significant decrease in PWV in the first 6 months of treatment $(P=0.009)$. Although a gradual increase in PWV was observed from 6 to 24 months $(P=0.001)$, it remained below baseline values at 24 months.

Patients in the HT group exhibited a significant decrease in PWV after 6 months of treatment with CPAP (1538.3 \pm 209.4 $\left.1489.7 \pm 204.2 \mathrm{~cm} \mathrm{~s}^{-1} ; P=0.005\right)$ and significant decreases were also found for BMI $\left(30.3 \pm 5.5-29.7 \pm 5.5 \mathrm{~kg} \mathrm{~m}^{-2} ; P<0.001\right)$, body weight $(88.2 \pm 18.3-86.3 \pm 18.2 \mathrm{~kg} ; P<0.001), P B F(30.7 \pm 7.1-29.9 \pm 6.3 \%$; $P=0.030)$, SBP $(146.3 \pm 15.3-140.5 \pm 17.3 \mathrm{~mm} \mathrm{Hg} ; P<0.001), \quad \mathrm{DBP}$ $(89.8 \pm 11.3-85.5 \pm 11.3 \mathrm{~mm} \mathrm{Hg} ; \quad P<0.001)$ and $\mathrm{HR}(81.4 \pm 12.8-$ $73.8 \pm 11.6$ b.p.m; $P<0.001)$ after 24 months of treatment (Figure 1). In comparison, patients in the NT group exhibited significant decreases in HR after 6 and 24 months of treatment $(79.7 \pm 13.3$ $72.3 \pm 10.3 ; P<0.001$, and $79.7 \pm 13.3-70.2 \pm 11.7 ; P<0.001$, respectively), as well as a significant decrease in PWV at 6 months $\left(1362.9 \pm 160.0-1323.2 \pm 152.1 \mathrm{~cm} \mathrm{~s}^{-1} ; \quad P=0.010\right)$. Although PWV increased gradually in the NT group from 6 to 24 months (1323.2 $\left.\pm 152.1-1359.4 \pm 161.4 \mathrm{~cm} \mathrm{~s}^{-1} ; P=0.022\right)$, values at 24 months remained below baseline (Figure 2).

\section{Parameters related to changes in PWV}

Results of stepwise multivariate regression analyses are summarized in Table 3. Age $(P=0.008)$, decreases in DBP $(P<0.001)$ and $\mathrm{HR}$ $(P<0.001)$ and higher initial serum levels of HDL-C $(P=0.040)$ were identified as independent factors contributing to decreases in PWV after 24 months of CPAP treatment across the total study population. Furthermore, a significant correlation was found between $\Delta$ PWV24 and $\triangle$ PWV6 $(r=0.517, P<0.001)$.

\section{DISCUSSION}

To our knowledge, this study is the first long-term observation to evaluate the effects of CPAP on arterial stiffness in OSA patients prospectively. In both the NT and HT groups, CPAP treatment decreased PWV in the first 6 months of treatment.

This study also evaluated various clinical parameters in both NT and HT OSA patients and the effects of CPAP treatment on these parameters. Patients in the HT group were more obese and had higher PWV and serum triacylglycerol levels compared with those in the NT group. Over the 2-year course of CPAP treatment, BMI, HR and blood pressure (SBP and DBP) level decreased continuously in HT patients.

Previous studies reported decreases in PWV in NT OSA patients over a 3- to 4-month observation period. ${ }^{11-15}$ In the present 
Table 1 Patient characteristics

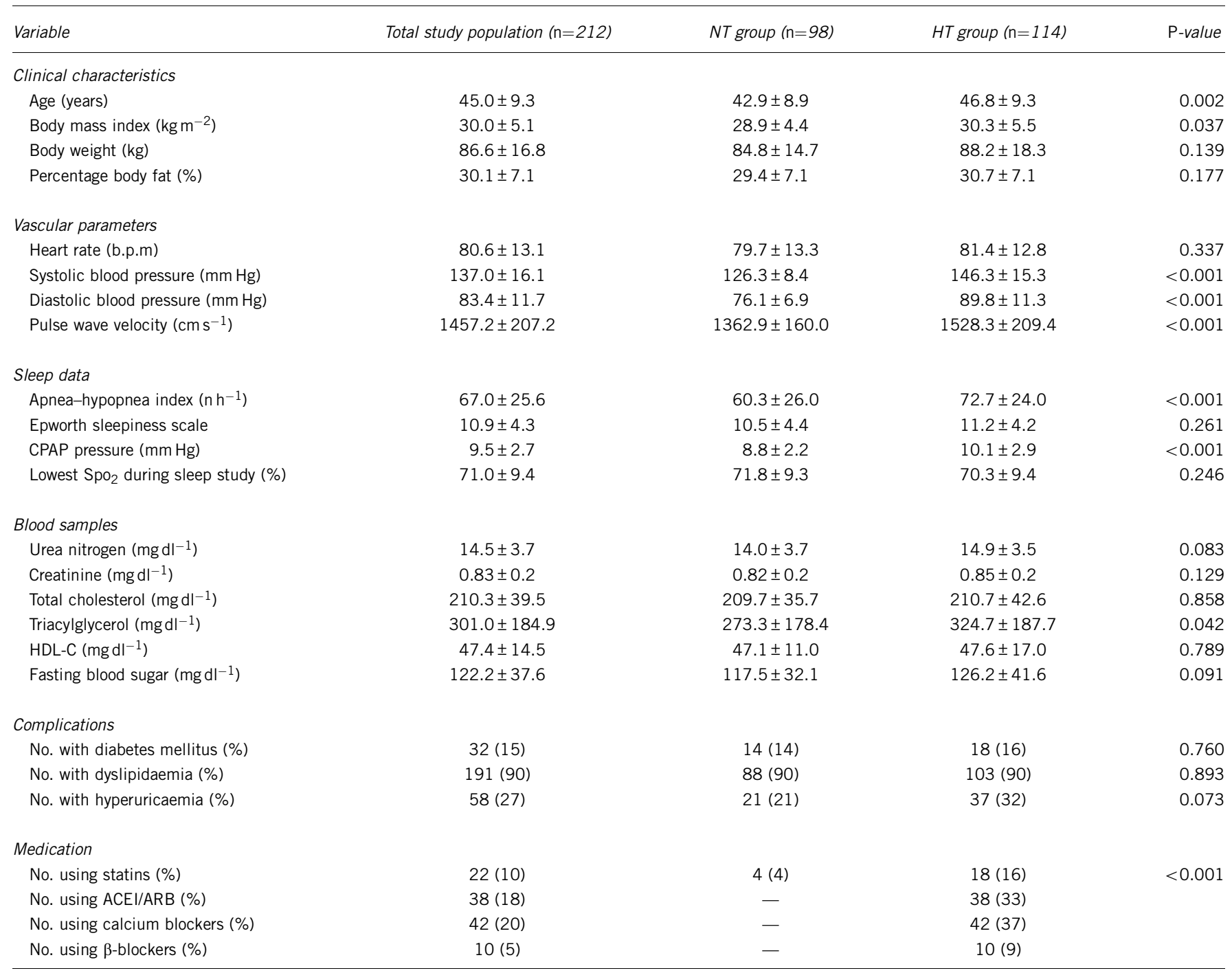

Abbreviations: ACEI, angiotensin-converting enzyme inhibitor; ARB, angiotensin receptor blocker; b.p.m, beats per minute; CPAP, continuous positive airway pressure; HDL-C, high-density lipoprotein-cholesterol; HT, hypertensive; NT, normotensive.

Unless indicated otherwise, data are given as the mean \pm s.d.

Table 2 Change in clinical parameters over 24 months treatment with continuous positive airway pressure

\begin{tabular}{|c|c|c|c|c|}
\hline \multirow[b]{2}{*}{ Total study population $(\mathrm{n}=212)$} & \multirow[b]{2}{*}{ Baseline } & \multicolumn{3}{|c|}{ Duration of CPAP treatment } \\
\hline & & 6 months & 12 months & 24 months \\
\hline Body mass index $\left(\mathrm{kg} \mathrm{m}^{-2}\right)^{\ddagger \ddagger \ddagger}$ & $29.7 \pm 5.1$ & $29.3 \pm 4.9 * *$ & $29.2 \pm 4.9 * * *$ & $29.2 \pm 5.1^{* * *}$ \\
\hline Body weight $(\mathrm{kg})^{\ddagger \ddagger}$ & $86.6 \pm 16.8$ & $85.7 \pm 16.4$ & $85.7 \pm 18.1$ & $85.2 \pm 16.6^{* *}$ \\
\hline Percentage body fat $(\%)^{\ddagger}$ & $30.1 \pm 7.1$ & $29.6 \pm 6.2$ & $29.5 \pm 6.6^{*}$ & $29.3 \pm 6.6^{* * *}$ \\
\hline Heart rate (b.p.m) & $80.6 \pm 13.1$ & $73.2 \pm 11.5^{* * *}$ & $74.3 \pm 11.8^{* * *}$ & $72.2 \pm 11.8^{* * *}$ \\
\hline Systolic blood pressure $(\mathrm{mm} \mathrm{Hg})^{\ddagger \ddagger}$ & $137.0 \pm 16.1$ & $135.6 \pm 16.6$ & $136.3 \pm 16.8$ & $133.6 \pm 16.6 * *$ \\
\hline Diastolic blood pressure $(\mathrm{mm} \mathrm{Hg})^{\ddagger \ddagger}$ & $83.4 \pm 11.7$ & $82.8 \pm 11.2$ & $83.1 \pm 11.5$ & $81.1 \pm 11.1 * *$ \\
\hline Pulse wave velocity $\left(\mathrm{cm} \mathrm{s}^{-1}\right)^{+\frac{1+1}{+}}$ & $1456.6 \pm 211.4$ & $1409.8 \pm 206.4^{* * *}$ & $1428.0 \pm 222.6^{*}$ & $1448.8 \pm 231.9$ \\
\hline
\end{tabular}

Abbreviations: b.p.m, beats per minute; CPAP, continuous positive airway pressure.

Data are presented as the mean \pm s.d. ${ }^{*} P<0.05$, ${ }^{* *} P<0.01,{ }^{* * *} P<0.001$ compared with baseline; ${ }^{\dagger} P<0.05,{ }^{\dagger} P<0.01,{ }^{\dagger+} P<0.001$ compared with 6 months of continuous positive airway pressure (CPAP) treatment; ${ }^{\ddagger} P<0.05,{ }^{\ddagger \ddagger} P<0.01,{ }^{\ddagger \ddagger} \neq P<0.001$ compared with changeover (ANOVA)

study, PWV in both NT and HT patients decreased initially before increasing gradually: during the initial 6 months of treatment, PWV decreased significantly and then increased gradually over the next 18 months. Despite the gradual increase in PWV over the final 18 months of treatment, PWV after 2 years of CPAP in both groups remained below baseline values. Therefore, we thought 

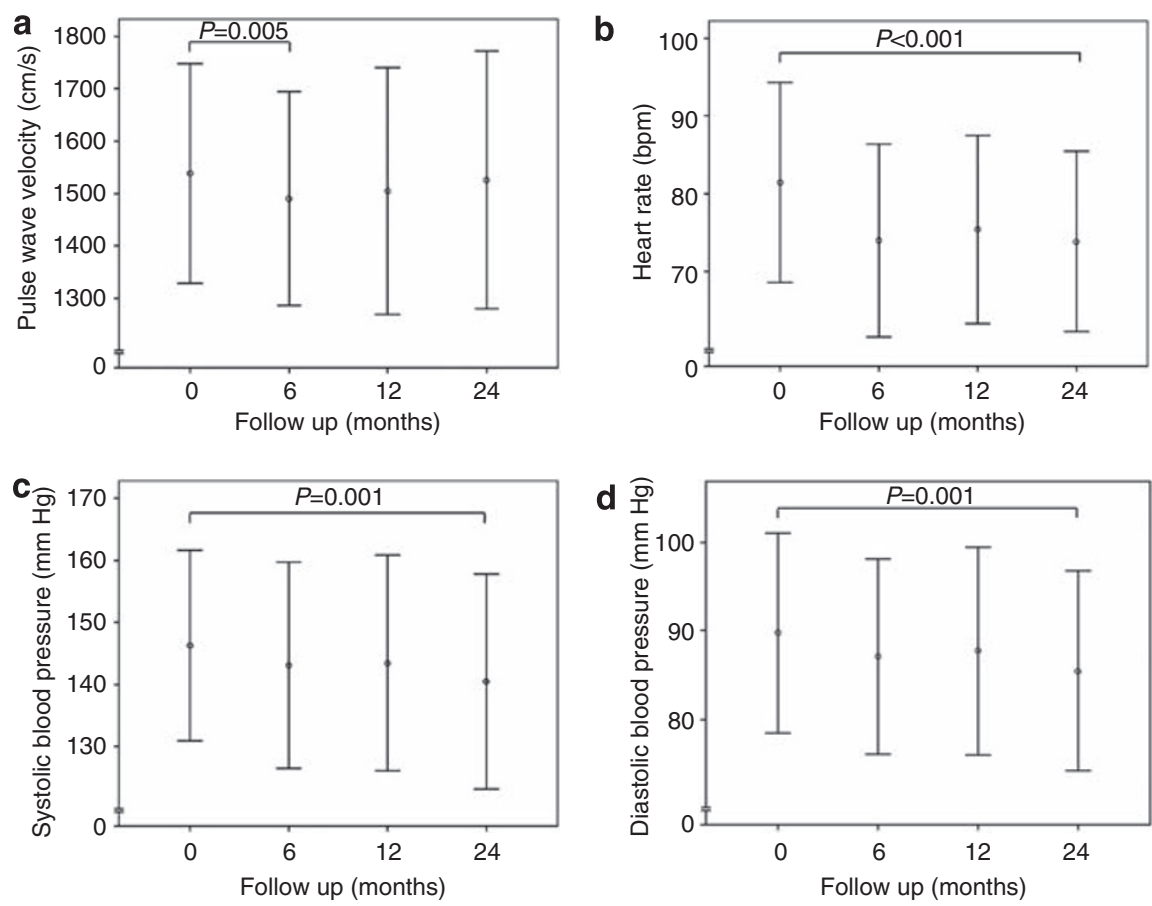

Figure 1 Effect of continuous positive airway pressure (CPAP) on clinical parameters in hypertensive (HT) patients. (a) Pulse wave velocity decreased initially in HT patients (from baseline to 6 months), but increased gradually thereafter in the cohort as a whole. In HT patients, 2 years of CPAP treatment decreased (b) heart rate, (c) systolic blood pressure and (d) diastolic blood pressure levels. Results are presented as mean \pm s.d. Probability values refer to comparisons of time-course curves by ANOVA for repeated measures.
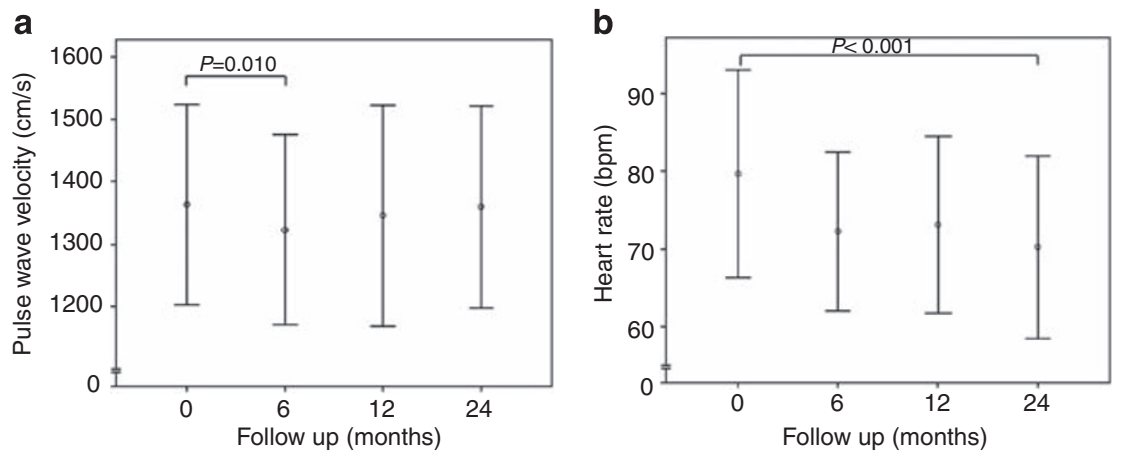

C

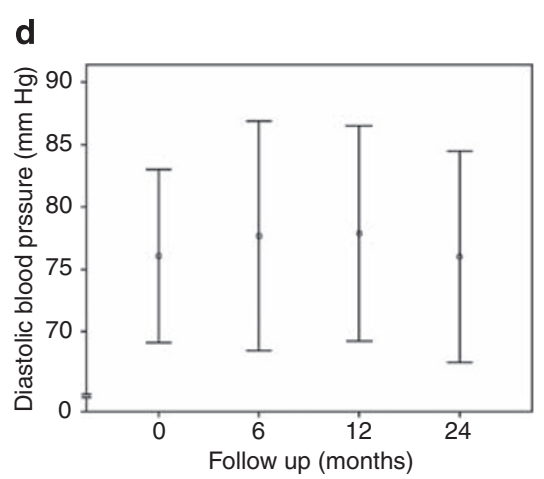

Figure 2 Effect of continuous positive airway pressure (CPAP) on clinical parameters in normotensive (NT) patients. (a) Pulse wave velocity decreased initially in NT patients (from baseline to 6 months), but increased gradually thereafter in the cohort as a whole. In NT patients, 2 years of CPAP treatment decreased (b) heart rate, but significant changes in (c) systolic blood pressure and (d) diastolic blood pressure were not seen. Results are presented as mean \pm s.d. Probability values refer to comparisons of time-course curves by ANOVA for repeated measures.

that the major effect of CPAP treatment appears within the first 6 months and the effect of CPAP on PWV in successive months is less.
The increase in PWV from 6 to 24 months coincides with the natural age-related progression in arterial stiffness and is consistent with the results of a previous study showing increases in 
Table 3 Regression coefficients (B) and their standard errors (s.e.), standardized coefficients $(\boldsymbol{\beta})$ and $\boldsymbol{P}$ values after multivariate regression analysis evaluating the association between changes in pulse wave velocity after 6 and 24 months treatment and various parameters

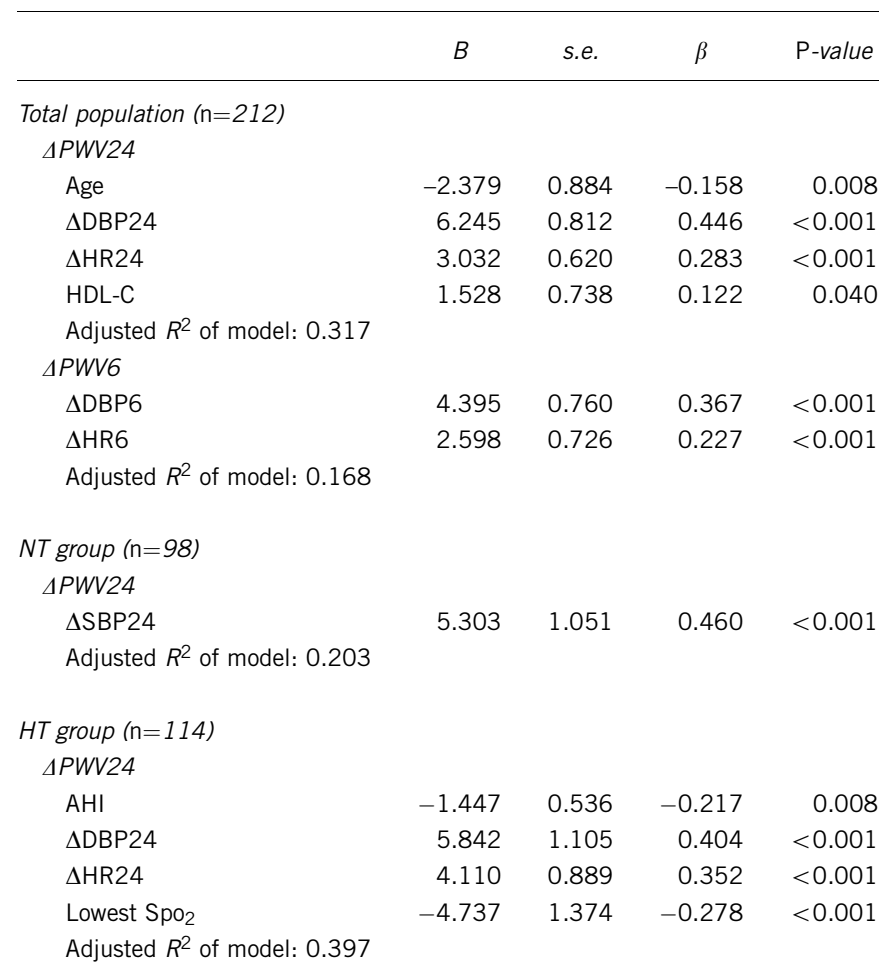

Abbreviations: AHI, apnea-hypopnea index; B, regression coefficients; CPAP, continuous positive airway pressure; HDL-C, high-density lipoprotein-cholesterol; HT, hypertensive; NT, normotensive; PWV, pulse wave velocity; $\beta$, standardized coefficient.

Changes in heart rate (HR), systolic (SBP) and diastolic blood pressure (DBP) were calculated as: $\Delta=$ baseline value - value after 24 months CPAP treatment.

carotid-femoral PWV over a 6-year period without any specific intervention. ${ }^{18}$ Thus, the improvement in PWV during the present study suggests that CPAP treatment ameliorates arterial stiffness. This is particularly true for the HT group in the present study, given that Benetos et al. ${ }^{18}$ have reported that PWV deteriorates over time in HT patients.

Chronic intermittent hypoxia has been linked with the progression of arterial stiffness in patients with OSA. Several experimental and clinical studies have shown that nocturnal hypopnea induces increases in blood pressure levels ${ }^{6,19}$ and the progression of arterial stiffness. ${ }^{18,19}$ Endothelial dysfunction ${ }^{4,20-22}$ and disturbances of sympathetic activity ${ }^{5,23}$ are recognized clinical features in OSA patients that increase vascular tone and accelerate the progression of arterial stiffness. ${ }^{7}$ Chronic intermittent hypoxia causes dyslipidaemia and initiates the development of atherosclerosis, both of which contribute to arterial stiffness. ${ }^{24}$ Decreasing nocturnal apnea-hypopnea by CPAP treatment may protect OSA patients from the progression of arterial stiffness, as well as decreasing blood pressure in these patients.

The HR of all OSA patients in the present study decreased continuously over the 2 years of treatment. Improvements in nocturnal hypoxia and sympathetic activity probably underlie the CPAPinduced reductions in HR. Chronic intermittent hypoxia is associated with hypoxemia, leading to decreased $\mathrm{O}_{2}$ saturation. An experimental study using mice suggested that chronic intermittent hypoxia itself induces sympathetic hyperactivation, and thus increases HR. ${ }^{25}$ Ziegler et al., ${ }^{23}$ showed that CPAP treatment for 10 days lowered daytime HR, as well as plasma and urine noradrenaline. In another study, ${ }^{26} \mathrm{CPAP}$ treatment for 3-4 days also reduced HR in OSA patients. The present study has shown, for the first time, that long-term CPAP treatment also improves HR. It seems likely that the effect of CPAP on HR could result from improvements in chronic intermittent hypoxia and suppression of sympathetic hyperactivation.

Multivariate regression analysis implicated age, decreases in DBP and HR and higher initial serum levels of HDL-C as independent factors contributing to the decrease in PWV across the entire study cohort. Experimental and clinical studies of PWV under conditions not affected by sympathetic tone indicate that PWV increases as a result of increases in HR. ${ }^{27,28}$ For example, PWV was higher in sympathectomized rats with a higher HR than in rats with a lower $\mathrm{HR}^{27}$ In addition, PWV was shown to increase in patients implanted with a pacemaker with increasing HR, measured at five different pacing frequencies. ${ }^{28}$ The correlation between HR variation and arterial stiffness was attributed to a mechanism whereby the increase in HR shortened the time available for recoil, thereby inducing arterial stiffening. The results of the present study suggest that the decrease in HR in OSA patients reduced PWV over the 2 years of treatment with CPAP.

It is known that high blood pressure worsens arterial stiffness. ${ }^{9}$ Arterial pressure is related directly to peripheral resistance and arterial caliber, and PWV is determined by the thickness of the arterial wall, as well as by arterial diameter. Arteries in patients with a higher DBP are under constant strong tension. This results in a decrease in arterial wall compliance and worsens arterial stiffness.

HDL-C prevents the progression of atherosclerosis by mediating the uptake of peripheral cholesterol and returning it to the liver for excretion in the bile and through the gut. ${ }^{29}$ Recent studies have investigated the contribution of the endothelial, antiapoptotic, antithrombotic, antioxidant and anti-inflammatory effects of HDL-C to its antiatherosclerotic action. ${ }^{30}$ This study suggests that CPAP treatment is likely to result in potentially greater improvements in arterial stiffness in OSA patients with high HDL-C levels. Incidentally, there is no relationship between improvements in PWV and statin use.

One limitation of this study is the lack of a control group consisting of patients with OSA who did not undergo CPAP treatment. All patients in the present study suffered from severe OSA that required CPAP treatment; given that OSA is a major risk factor for cardiovascular diseases, ${ }^{1,2}$ it was not possible to include an untreated control group in the present study. Therefore, when interpreting the results of this study, we used the findings reported by Benetos et al. ${ }^{18}$ as an indicator of the natural course of PWV progression, despite differences between measures of brachial-ankle PWV (the present study) and carotid-femoral PWV (Benetos et al. ${ }^{18}$ ).

Another limitation of this study is the question regarding the validity of $\triangle \mathrm{PWV} 24$ as a marker with which to assess reductions in PWV. In all patients in this study, PWV decreased during the first 6 months of treatment, then increased gradually over the next 18 months. This biphasic response of PWV complicated estimations of the long-term improvements in PWV. We propose that CPAP treatment improves arterial stiffness in the initial 6 months and that the following gradual increases in PWV may reflect the natural progression in PWV. This notion is supported by our findings of a significant correlation between $\triangle \mathrm{PWV} 6$ and $\triangle \mathrm{PWV} 24$. Thus, it seems that $\triangle \mathrm{PWV} 24$ is an appropriate indicator of the long-term (over 2 years) effect of CPAP treatment in OSA patients.

In addition, one more limitation of this study is the absence of precise data regarding CPAP adherence. Although providing data 
regarding CPAP adherence overtime is an important point that should influence PWV values, we could not obtain precise usage time of CPAP, except for the confirmation of $5 \mathrm{~h}$ CPAP treatment.

In conclusion, we found a significant decrease in PWV in both NT and HT patients after 6 months of CPAP treatment. In HT patients, long-term CPAP treatment significantly decreases blood pressure, which may help to explain PWV improvement.

\section{ACKNOWLEDGEMENTS}

We thank Dr Tetsu Yamaguchi, President of Toranomon Hospital and Dr Yasumichi Mori, Director of the Metabolism Department of Toranomon Hospital, for their contributions to data analyses.

Author Contributions: Study concept and design: Tsunenori Saito, Tsunehiro Saito and Mizuno. Acquisition of data: Tsunenori Saito, Tsunehiro Saito and Sugiyama. Analysis and interpretation of data: Tsunenori Saito, Sugiyama and Asai. Drafting of the paper: Tsunenori Saito and Mizuno. Critical revision of the paper for important intellectual content: Tsunehiro Saito, Asai and Yasutake. Administrative, technical and material support: Tsunehiro Saito and Sugiyama.

1 Peppard PE, Young T, Palta M, Skatrud J. Prospective study of the association between sleep-disordered breathing and hypertension. N Engl J Med 2000; 342: 1378-1384

2 Shahar E, Whitney CW, Redline S, Lee ET, Newman AB, Javier Nieto F, O'Connor GT, Boland LL, Schwartz JE, Samet JM. Sleep-disordered breathing and cardiovascular disease: cross-sectional results of the Sleep Heart Health Study. Am J Respir Crit Care Med 2001; 163: 19-25.

3 Yokoe T, Minoguchi K, Matsuo H, Oda N, Minoguchi H, Yoshino G, Hirano T, Adachi M. Elevated levels of C-reactive protein and interleukin- 6 in patients with obstructive sleep apnea syndrome are decreased by nasal continuous positive airway pressure. Circulation 2003; 107: 1129-1134

4 Ip MS, Tse HF, Lam B, Tsang KW, Lam WK. Endothelial function in obstructive sleep apnea and response to treatment. Am J Respir Crit Care Med 2004; 169: 348-353.

5 Hedner J, Darpö B, Ejnell H, Carlson J, Caidahl K. Reduction in sympathetic activity after long-term CPAP treatment in sleep apnoea: cardiovascular implications. Eur Respir J 1995; 8: 222-229.

6 Chin K, Nakamura T, Takahashi K, Sumi K, Matsumoto H, Niimi A, Fukuhara S, Mishima M, Nakamura T. Falls in blood pressure in patients with obstructive sleep apnoea after long-term nasal continuous positive airway pressure treatment. $J$ Hypertens 2006; 24: 2091-2099.

7 Jelic S, Bartels MN, Mateika JH, Ngai P, DeMeersman RE, Basner RC. Arterial stiffness increases during obstructive sleep apneas. Sleep 2002; 25: 850-855.

8 Drager LF, Bortolotto LA, Lorenzi MC, Figueiredo AC, Krieger EM, Lorenzi-Filho G. Early signs of atherosclerosis in obstructive sleep apnea. Am J Respir Crit Care Med 2005; 172: 613-618.

9 Asmar R, Benetos A, Topouchian J, Laurent P, Pannier B, Brisac AM, Target R, Levy BI. Assessment of arterial distensibility by automatic pulse wave velocity measurement: validation and clinical application studies. Hypertension 1995; 26: 485-490.

10 Laurent S, Boutouyrie P, Asmar R, Gautier I, Laloux B, Guize L, Ducimetiere P, Benetos A. Aortic stiffness is an independent predictor of all-cause and cardiovascular mortality in hypertensive patients. Hypertension 2001; 37: 1236-1241.
11 Drager LF, Bortolotto LA, Figueiredo AC, Krieger EM, Lorenzi GF. Effects of continuous positive airway pressure on early signs of atherosclerosis in obstructive sleep apnea. Am J Respir Crit Care Med 2007; 176: 706-712.

12 Kitahara Y, Hattori N, Yokoyama A, Nakajima M, Kohno N. Effect of CPAP on brachialankle pulse wave velocity in patients with OSAHS: an open-labelled study. Respir Med 2006; 100: 2160-2169.

13 Kuramoto E, Kinami S, Ishida Y, Shiotani H, Nishimura Y. Continuous positive nasal airway pressure decreases levels of serum amyloid $A$ and improves autonomic function in obstructive sleep apnea syndrome. Int J Cardiol 2009; 135: 338-345.

14 Shiina K, Tomiyama H, Takata Y, Yoshida M, Kato K, Saruhara H, Hashimura Y, Matsumoto C, Asano K, Usui $Y$, Yamashina A. Effect of CPAP therapy on the sympathovagal balance and arterial stiffness in obstructive sleep apnea. Respir Med 2010; 104: 911-916.

15 Chung S, Yoon IY, Lee CH, Kim JW. The effects of nasal continuous positive airway pressure on vascular function and serum cardiovascular risk factors in obstructive sleep apnea syndrome. Sleep Breath 2010 (e-pub ahead of print 19 January 2010).

16 Ymamashina A, Tomiyama H, Takeda K, Tsuda H, Arai T, Hirose K, Koji Y, Hori S, Yamamoto Y. Validity, reproducibility, and clinical significance of noninvasive brachial-ankle pulse wave velocity measurement. Hypertens Res 2002; 25: 359-364.

17 Johns MW. A new method for measuring daytime sleepiness: the Epworth sleepiness scale. Sleep 1991; 14: 540-545.

18 Benetos A, Adamopoulos C, Bureau JM, Temmar M, Labat C, Bean K, Thomas F, Pannier B, Asmar R, Zureik M, Safar M, Guize L. Determinants of accelerated progression of arterial stiffness in normotensive subjects and in treated hypertensive subjects over a 6-year period. Circulation 2002; 105: 1202-1207.

19 Fletcher EC, Bao G, Miller III CC. Effect of recurrent episodic hypocapnic, eucapnic, and hypercapnic hypoxia on systemic blood pressure. J Appl Physiol 1995; 78: 1516-1521.

20 Schulz R, Mahmoudi S, Hattar K, Sibelius U, Olschewski H, Mayer K, Seeger W, Grimminger $F$. Enhanced release of superoxide from polymorphonuclear neutrophils in obstructive sleep apnea: impact of continuous positive airway pressure therapy. $A m$ J Respir Crit Care Med 2000; 162: 566-570.

21 Lattimore JL, Wilcox I, Skilton M, Langenfeld M, Celermajer DS. Treatment of obstructive sleep apnoea leads to improved microvascular endothelial function in the systemic circulation. Thorax 2006; 61: 491-495.

22 El Solh AA, Akinnusi ME, Baddoura FH, Mankowski CR. Endothelial cell apoptosis in obstructive sleep apnea: a link to endothelial dysfunction. Am J Respir Crit Care Med 2007; 175: 1186-1191.

23 Ziegler MG, Mills PJ, Loredo JS, Ancoli-Israel S, Dimsdale JE. Effect of continuous positive airway pressure and placebo treatment on sympathetic nervous activity in patients with obstructive sleep apnea. Chest 2001; 120: 887-893.

24 Savransky V, Nanayakkara A, Li J, Bevans S, Smith PL, Rodriguez A, Polotsky VY. Chronic intermittent hypoxia induces atherosclerosis. Am J Respir Crit Care Med 2007; 175: 1290-1297.

25 Dematteis M, Julien C, Guillermet C, Sturm N, Lantuejoul S, Mallaret M, Lévy P, Gozal $\mathrm{E}$. Intermittent hypoxia induces early functional cardiovascular remodeling in mice. $\mathrm{Am}$ J Respir Crit Care Med 2008; 177: 227-235.

26 Sumi K, Chin K, Takahashi K, Nakamura T, Matsumoto H, Niimi A, Mishima M. Effect of nCPAP therapy on heart rate in patients with obstructive sleep apnea-hypopnea. QJM 2006; 99: 545-553.

27 Mircoli L, Mangoni AA, Giannattasio C, Mancia G, Ferrari AU. Heart rate-dependent stiffening of large arteries in intact and sympathectomized rats. Hypertension 1999; 34: 598-602.

28 Lantelme P, Mestre C, Lievre M, Gressard A, Milon H. Heart rate: an important confounder of pulse wave velocity assessment. Hypertension 2002; 39: 1083-1087.

29 Ross R, Glomset JA. Atherosclerosis and the arterial smooth muscle cell: proliferation of smooth muscle is a key event in the genesis of the lesions of atherosclerosis. Science 1973; 180: 1332-1339.

30 Mineo C, Deguchi H, Griffin JH, Shaul PW. Endothelial and antithrombotic actions of HDL. Circ Res 2006; 98: 1352-1364. 